\title{
An Optimized Scheme to Resist Primary User Emulation Attacks
}

\author{
Jie Xiao, Yuebin Chen, Chuanxi Xing, Jiangfeng Yang and Zhenling Yang \\ School of Electrical and Information Technology, Yunnan Minzu University, Kunming, Yunnan, China
}

\begin{abstract}
The problem of cognitive radio (CR) network security has become an important problem in cognitive radio technology. One of the most common attacks is primary user emulation attacks (PUEA), where some malicious users simulate CR users to prevent them from accessing the vacant frequency. Here, a cooperative spectrum sensing (CSS) method with optimal weight coefficient based on improved energy detection is proposed. In the proposed scheme, we combine sensing information using the optimal weight coefficient at a fusion center and apply the improved energy detection, which effectively prevent the PUEA. Simulation results show that the method outperforms the traditional energy detection with optimal weight coefficient in detection performance.
\end{abstract}

Keywords-cognitive radio; cooperative spectrum sensing; primary user emulation attack; energy detection; optimal weight coefficient

\section{INTRODUCTION}

With the development of wireless communication technology, spectrum resources become so deficient. Hence, CR network has been widely adopted as a promising technology to overcome the spectrum scarcity by CR users (CUs) to operate opportunistically in the idle space of the licensed bands in the co-existence of the primary users (PUs) [1]. Spectrum sensing is a fundamental functionality of CR network [2]. In the spectrum sensing process, it will produce a variety of security issues, which the most common one is the primary user emulation attacks (PUEA). The PUEA is that when the malicious users (MU) find idle spectrum, it will send a similar signal with the primary user's signal to prevent the CU to detect the idle spectrum. Recently, in [3] and [4], the authors classify $\mathrm{CR}$ attacks and provide some contributions on security threats, detection techniques, new challenges and future research directions. In order to solve this problem, cooperative spectrum sensing (CSS) technology is put forward At the same time, cooperative spectrum sensing is a method which can improve the detection performance effectively [5].

Typically, the primary user and the CR users are separate to each other. CR users can not accurately know all information of the primary user, so we usually use energy detection. In [6], a soft combing method, equivalent to maximal ratio combining (MRC), is developed where the sensing statistics of different CR users are combined by using the optimal weight coefficient. In [7], the cooperative spectrum sensing scheme with optimal weight coefficient based on energy detection is proposed. Compared with the traditional MRC method, the method proposed in [7] improves the detection probability. Based on the research of [7], this paper propose an improved energy detection, which can be more effective than the method in [7].

In this paper, we set up a cooperative spectrum sensing model about the presence of PUEA and propose a scheme to maximize the detection probability of primary user in the cognitive radio networks. Each CR user of CR networks sends the received information to the fusion center and is combined with the optimal weight coefficient. Finally, according to the Neyman-Pearson criterion we can make the final decision. In this paper, the maximum detection probability of the primary user is obtained by deriving the optimal weight coefficient. Therefore, this scheme can effectively resist PUEA and improve the detection performance of the system.

The rest of this letter is organized as follows. Section II introduces the system model of cooperative spectrum sensing with the presence of PUEA. In Section III, the optimal weight coefficients based on improved energy detection in the presence of PUEA is derived and the maximum detection probability of primary user is calculated. Discussion along with comparative simulation results is presented in Section IV.

\section{SYSTEM MODEL}

In this model, we consider cooperative spectrum sensing in a CR network having N CR users coexisting with a malicious user to detect the presence of one primary transmitter, as shown in Figure I.

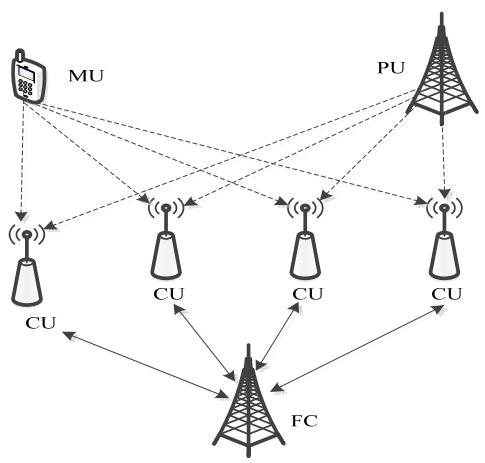

FIGURE I. SYSTEM MODEL OF THE COGNITIVE RADIO NETWORK

In the entire CR network, we assume that PUEA always exists. So, there are two kinds of signal received by the $i$ th $(1 \leq i \leq N)$ CR user at the $t$ th time instant. 


$$
\begin{array}{lr}
y_{i 1}(t)=h_{m i}(t) x_{m}(t)+n_{i}(t) & H_{0} \\
y_{i 2}(t)=h_{p i}(t) x_{p}(t)+h_{m i}(t) x_{m}(t)+n_{i}(t) & H_{1}
\end{array}
$$

where $H_{0}$ is the situation at the absence of primary user and $H_{1}$ is the situation at the presence of primary user. $h_{p i}(t)$ and $h_{m i}(t)$ denote the instantaneous channel gain between primary user and $i$ th CR user and between malicious user and $i$ th CR user respectively. $x_{p}(t)$ and $x_{m}(t)$ are the signal transmitted by primary user and malicious user. $n_{i}(t)$ is the additive white Gaussian noise at the $i$ th the CR user with zero mean and variance $\sigma_{n}{ }^{2}$.

In cooperative spectrum sensing, each CR user will send the local decision results to the fusion center, and then the fusion center makes a final decision that PU is presence or not [2]. Under normal conditions, fusion method is mainly divided into two kinds of hard fusion and soft fusion. In this paper, we mainly use the soft fusion method, which the signals received by CR user are weighted by some coefficients $w_{i}(i=1,2 \ldots N)$ and fused to make a final decision in fusion center [6].

The assumption is that the channel from the CR user to the fusion center is perfect. The combined signal in the fusion center at the $t$ th time instant is

$$
y(t)= \begin{cases}\sum_{i=1}^{N} w_{i} y_{i 1}(t) & H_{0} \\ \sum_{i=1}^{N} w_{i} y_{i 2}(t) & H_{1}\end{cases}
$$

In this paper, we adopt the improved energy detection method in which $M$ samples of $y(t)$ are summed during one detection interval.

$$
Y=\sum_{t=1}^{M} \mid y\left(\left.t\right|^{p}\right.
$$

The fusion center then calculates the decision statistic $Y$ for each detection interval to make a global decision.

\section{COOPERATIVE SPECTRUM SENSING UNDER IMPROVED ENERGY DETECTION}

In this section, we will derive the optimal weight coefficients based on the improved energy detection to maximize the detection probability of primary user in the presence of PUE attacks. Comparing the statistic $Y$ with the threshold value, the false alarm probability $P_{f}$ and detection probability $P_{d}$ over a detection interval are defined as [8],

$$
p_{f}=\left(Y \geq \lambda \mid H_{0}\right)
$$

$$
p_{d}=\left(Y \geq \lambda \mid H_{1}\right)
$$

where $\lambda$ in (4) and (5) is a detection threshold value in improved energy detection.

Because of using the Neyman-Pearson criterion, we need to give a value of $P_{f}$ in advance. For a given $P_{f}$ and soft fusion method, the most important problem is to find out the optimal weight coefficient $W_{\text {opt }}=\left[w_{1 o p t}, w_{2 o p t}, \ldots w_{\text {Nopt }}\right]$ to maximize the detection probability. Among this, $W=\left[w_{1}, w_{2}, \ldots w_{N}\right]$ is a vector of weight coefficients for the combination at the fusion center.

In the actual cognitive radio network, the PUE attacker's signal is similar to the primary user's signal. Therefore, we assume that the primary signal $x_{p}(t)$ follows a complex Gaussian distribution, i.e. $x_{p}(t) \sim C N\left(0, \sigma_{P U}^{2}\right)$, the $x_{m}(t)$ follows the complex Gaussian distribution, i.e. $x_{m}(t) \sim C N\left(0, \sigma_{P U E A}^{2}\right)$. For given $h_{p i}$ and $h_{m i}$, the combined signal $y(t)$ is also a complex Gaussian distributed random variable,

$$
y(t) \sim \begin{cases}C N\left(0, \sigma_{0}^{2}\right) & H_{0} \\ C N\left(0, \sigma_{1}^{2}\right) & H_{1}\end{cases}
$$

where, $\sigma_{0}^{2}$ and $\sigma_{1}^{2}$ are the variance of $y(t)$ for $H_{0}$ and $H_{1}$ respectively.

$$
\sigma_{0}^{2}=\sigma_{\text {PUEA }}^{2}\left|\sum_{i=1}^{N} w_{i} h_{m i}\right|^{2}+\sigma_{n}^{2} \sum_{i=1}^{N}\left|w_{i}\right|^{2}
$$

$$
\sigma_{1}^{2}=\sigma_{P U}^{2}\left|\sum_{i=1}^{N} w_{i} h_{p i}\right|^{2}+\sigma_{P U E A}^{2}\left|\sum_{i=1}^{N} w_{i} h_{m i}\right|^{2}+\sigma_{n}^{2} \sum_{i=1}^{N}\left|w_{i}\right|^{2}
$$

Give

$$
H_{m}=\left[h_{m 1}(t), h_{m 2}(t), \ldots h_{m N}(t)\right]^{T}, H_{p}=\left[h_{p 1}(t), h_{p 2}(t), \ldots h_{p N}(t)\right]^{T}
$$

According to (7) and (8), $\sigma_{0}^{2}$ and $\sigma_{1}^{2}$ can be denoted,

$$
\sigma_{0}^{2}=\sigma_{P U E A}^{2} W H_{m} H_{m}{ }^{H} W^{H}+\sigma_{n}^{2} W W^{H}
$$

$$
\sigma_{1}^{2}=\sigma_{P U}^{2} W H_{p} H_{p}{ }^{H} W^{H}+\sigma_{P U E A}^{2} W H_{m} H_{m}{ }^{H} W^{H}+\sigma_{n}^{2} W W^{H}
$$

where, $H$ is the Hermitian transpose.

Based on the paper [9], the decision statistic $Y$ is similar to the gamma distribution by changing the mean and variance. So we have 


$$
p_{f}=\frac{\Gamma\left(k_{0}, \frac{\lambda}{\theta_{0}}\right)}{\Gamma\left(k_{0}\right)}
$$

$$
p_{d}=\frac{\Gamma\left(k_{1}, \frac{\lambda}{\theta_{1}}\right)}{\Gamma\left(k_{1}\right)}
$$

$$
\begin{aligned}
& \text { where, } k_{0}=k_{1}=M \frac{\Gamma^{2}\left(\frac{p+1}{2}\right)}{\Gamma\left(\frac{2 p+1}{2}\right) \sqrt{\pi}-\Gamma^{2}\left(\frac{p+1}{2}\right)} \\
& \theta_{0}=2^{\frac{p}{2}} \frac{\sqrt{\pi} \Gamma\left(\frac{2 p+1}{2}\right)-\Gamma^{2}\left(\frac{p+1}{2}\right)}{\Gamma\left(\frac{p+1}{2}\right) \sqrt{\pi}} \sigma_{0}^{p} \\
& \theta_{1}=2^{\frac{p}{2}} \frac{\sqrt{\pi} \Gamma\left(\frac{2 p+1}{2}\right)-\Gamma^{2}\left(\frac{p+1}{2}\right)}{\Gamma\left(\frac{p+1}{2}\right) \sqrt{\pi}} \sigma_{1}^{p}
\end{aligned}
$$

$\Gamma(\cdot)$ and $\Gamma(\cdot, \cdot)$ are Gamma function and upper incomplete Gamma function respectively.

Given $p_{f}=\varepsilon, \varepsilon \in[0,1]$, the decision threshold $\lambda$ is represented as,

$$
\lambda=\Gamma^{-1}\left(k_{0}, \varepsilon \cdot \Gamma\left(k_{0}\right)\right) \cdot \theta_{0}
$$

where $\Gamma^{-1}(\cdot$,$) is the inverse incomplete Gamma function [10].$ According to (12) and (13), $P_{d}$ can be rewritten as,

$$
p_{d}=\frac{\Gamma\left(k_{1}, \Gamma^{-1}\left(k_{0}, \varepsilon \Gamma\left(k_{0}\right)\right) \frac{\theta_{0}}{\theta_{1}}\right)}{\Gamma\left(k_{1}\right)}
$$

In this paper, $k_{0}=k_{1}$, for a given $M$, we can obtain the value of $k_{0}$. Thanks to the monotonicity of Gamma function, to maximize $P_{d}$ is equivalent to minimize $\theta_{0} / \theta_{1}$. According to (14) and (15),

$$
\frac{\theta_{0}}{\theta_{1}}=\left(\frac{\sigma_{0}^{2}}{\sigma_{1}^{2}}\right)^{\frac{p}{2}}
$$

According to (9) and (10),

$$
\begin{aligned}
\frac{\sigma_{0}{ }^{2}}{\sigma_{1}{ }^{2}} & =\frac{\sigma_{P U E A}^{2} W H_{m} H_{m}{ }^{H} W^{H}+\sigma_{n}^{2} W W^{H}}{\sigma_{P U}^{2} W H_{P} H_{P}{ }^{H} W^{H}+\sigma_{P U E A}^{2} W H_{m} H_{m}{ }^{H} W^{H}+\sigma_{n}^{2} W W^{H}} \\
& =\frac{1}{1+\frac{\sigma_{P U}^{2} W H_{p} H_{p}{ }^{H} W^{H}}{\sigma_{P U E A}^{2} W H_{m} H_{m}{ }^{H} W^{H}+\sigma_{n}{ }^{2} W W^{H}}}
\end{aligned}
$$

where $\sigma_{0}^{2} / \sigma_{1}^{2}<1$, when the value of $p$ increase, $\theta_{0} / \theta_{1}$ will reduce. Hence, we need to calculate the minimum value of $\sigma_{0}^{2} / \sigma_{1}^{2}$ when $p$ is established.

$$
\text { Let } \Phi=\frac{\sigma_{P U}^{2} W H_{p} H_{p}{ }^{H} W^{H}}{\sigma_{P U E A}^{2} W H_{m} H_{m}{ }^{H} W^{H}+\sigma_{n}^{2} W W^{H}} \text { to minimize }
$$

$\sigma_{0}^{2} / \sigma_{1}^{2}$ is equivalent to maximize $\Phi$. Take partial derivative of $\Phi$ with respect to $W$.

$$
\begin{array}{r}
{\left[2 \sigma_{P U}^{2} H_{p} H_{p}{ }^{H} W^{H}\left(\sigma_{P U E A}^{2} W H_{m} H_{m}{ }^{H} W^{H}+\sigma_{n}{ }^{2} W W^{H}\right)\right.} \\
\frac{\partial \Phi}{\partial W}=\frac{\left.-2\left(\sigma_{\text {PUEA }}^{2} H_{m} H_{m}{ }^{H} W^{H}+\sigma_{n}^{2} W^{H}\right) \sigma_{P U}{ }^{2} W H_{p} H_{p}{ }^{H} W^{H}\right]}{\left(\sigma_{\text {PUEA }}^{2} W H_{m} H_{m}{ }^{H} W^{H}+\sigma_{n}{ }^{2} W W^{H}\right)^{2}}
\end{array}
$$

Let $\frac{\partial \Phi}{\partial W}=0$, the optimal weight coefficient $W_{\text {opt }}$ is,

$$
W_{\text {opt }}=\left[\left(\sigma_{\text {PUEA }}^{2} H_{m} H_{m}{ }^{H}+\sigma_{n}^{2} I\right)^{-1} H_{p}\right]^{H}
$$

where $I$ is the identity matrix, and the minimal $\sigma_{0}^{2} / \sigma_{1}^{2}$,

$$
\left(\frac{\sigma_{0}^{2}}{\sigma_{1}^{2}}\right)_{\min }=\frac{1}{1+\sigma_{P U}^{2} H_{p}\left(\sigma_{P U E A}^{2} H_{m} H_{m}{ }^{H}+\sigma_{n}^{2} I\right)^{-1} H_{p}{ }^{H}}
$$

According to (18),

$$
\left(\frac{\theta_{0}}{\theta_{1}}\right)_{\min }=\left(\frac{1}{1+\sigma_{P U}^{2} H_{p}\left(\sigma_{P U E A}^{2} H_{m} H_{m}{ }^{H}+\sigma_{n}^{2} I\right)^{-1} H_{p}{ }^{H}}\right)^{\frac{p}{2}}
$$

using (17) and (23),

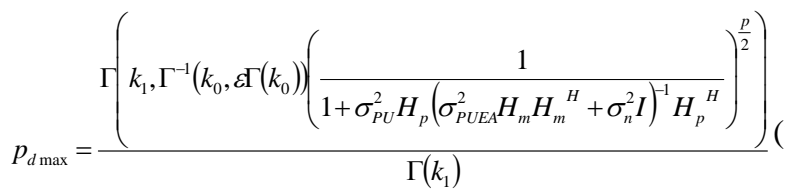




\section{Simulation RESUlts}

We implement the simulations of cooperative spectrum sensing scheme with the existence of PUEA. In this paper, there are one PUEA, one PU and $4 \mathrm{CU}$ in cognitive radio network. The number of samples within a detection interval is $\mathrm{M}=4$.

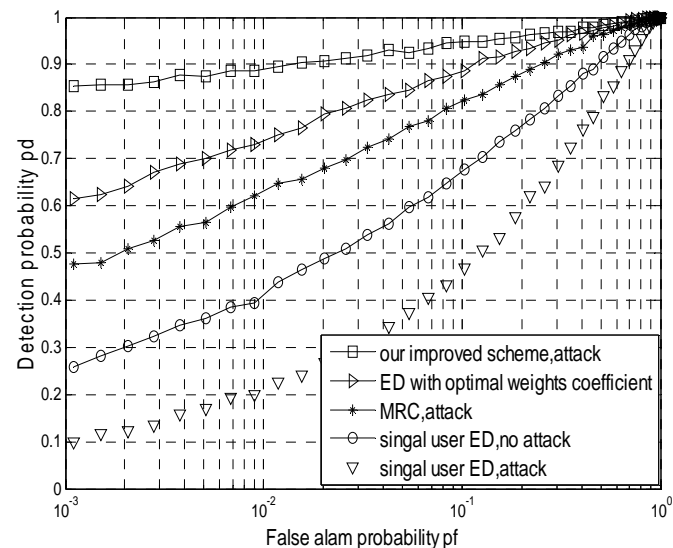

FIGURE II. THE ROC CURVE FOR OUR SCHEME, ENERGY DETECTION WITH OPTIMAL WEIGHT COEFFICIENT, CONVENTIONAL MRC AND SINGLE-USER ENERGY DETECTION.

Figure II display the ROC curve for our scheme, energy detection with optimal weight coefficient, conventional MRC and single-use energy detection scheme. In the simulation, all channel information are assumed to be known for the CR users. Assume $x_{p}(t) \sim C N(0,1), x_{m}(t) \sim C N(0,1)$ and the SNR between primary user and every CR user is $0 \mathrm{~dB}$, we can discuss them in the presence of PU EA. For conventional MRC, the weighting coefficients is $w_{i}=0.25(i=1,2,3,4)$.

The value of $p$ is 3 . From FIGURE II, we find that the detection probability of conventional MRC and single-user energy detection schemes are decline so severe under the effect of PUEA. In the case of the same false alarm probability, the detection probability of our scheme is better than that of energy detection with optimal weight coefficient. So our proposed scheme improves the detection performance of system.

Figure III describes the detection performance under different SNR at the presence of PUEA. In the simulation, given $p_{f}=0.1$, the SNR between primary user and CR users is same for each CR user. We defined,

$$
x=\frac{\sigma_{P U}^{2}}{\sigma_{P U E A}^{2}}
$$

$X$ reveals the strength of the attack. Smaller values of $x$ represent a strong attack. In Figure III, detection performance of the proposed scheme is compared with energy detection with the optimal weight coefficient scheme where $x$ is given as $0.1,1,10$ respectively in [7]. In the case of low SNR, the detection probability of our scheme is relatively high. At the same time, we find that the detection performance is improved effectively with the reduced of $x$ under the low SNR.

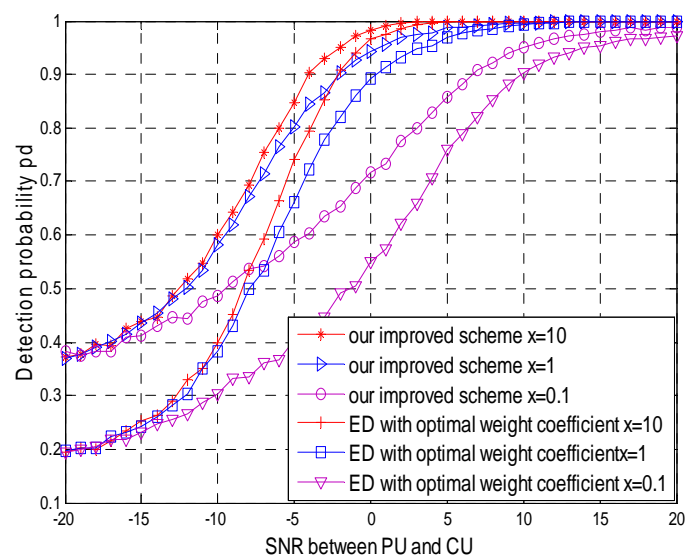

FIGURE III. DETECTION PROBABILITY VERSUS SNR FOR OUR SCHEME, ENERGY DETECTION WITH OPTIMAL WEIGHT COEFFICIENT, $p=3$.

Figure IV displays the detection probability with the change of SNR for our proposed scheme. According to (18) and (19), a large numerical value of $p$ indicates a small $\theta_{0} / \theta_{1}$ to make the detection probability bigger. In FIGURE IV, we assume that the value of $x$ is 0.1 and $p$ is set as $2,3,4,5$. With the increase of $p$, the probability of detection is gradually increased at the same SNR. But the magnitude of the increase is in constant decline. When $p$ is set to 10 , we can obtain the curve graph of the maximal detection probability. So assuming a stronger attack exist, we can improve the detection probability through increasing the value of $p$.

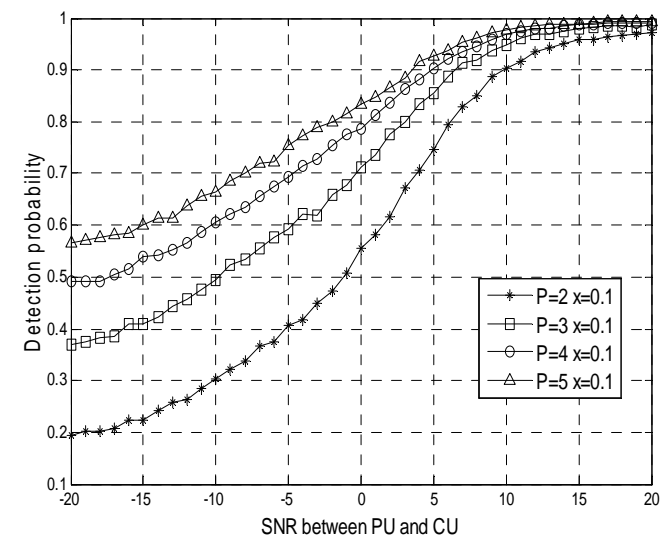

FIGURE IV. THE DETECTION PROBABILITY VERSUS SNR FOR DIFFERENT VALUE OF $p, x=0.1$

\section{ACKNOWLEDGMENT}

This work was partly supported by National Natural Science Foundation of China (NO.61261022) and innovation 
team project of Yunnan Minzu University. Yuebin Chen is the corresponding author.

\section{REFERENCES}

[1] S. Haykin, "Cognitive radio: brain-empowered wireless communications,” IEEE J. Sel. Areas Commun. Vol. 23, no. 2, pp.201220, Feb. 2005.

[2] MISHRA S M, SAHAI A, BROADERSON R W. "Cooperative sensing among cognitive radios,” [C]//ICC'06: IEEE International Conference Communications. Piscataway, NJ:IEEE, 2006:1658 -1663.

[3] Fragkiadakis AG,Tragos EZ, Askoxylakis IG, "Asurvery on security threats and detection techniques in cognitive radio network," IEEE Communications Survey and Tutorials 2013, 15: 428-445.

[4] Attar A, Tang H, Vasilakos AV, Yu FR, Leung VCM, "A survey of security challengs in cognitive radio networks:solution and future research directions,” Proceedings of the IEEE 2012, 100: 3172-3186.

[5] Zou Y L, Yao Y D, Zheng B Y. “Cooperative relay techniques for cognitive radio systems:spectrum sensing and secondary user transmissions[J],” Communications Magazine, 2012, 50:98-103

[6] J. Ma, G. Zhao, Y. Li. "Soft combination and detection for cooperative spectrum sensing in cognitive radio networks," IEEE Trans. Wireless Commun, Nov. 2008, vol. 7, no. 11, pp.4502-4507.

[7] Chen C, Cheng H B, Yao Y-D. "Cooperative spectrum sensing in cognitive radio networks in the presence of the primary user emulation attack[J].” IEEE Transactions on Wireless Communications 2011, 10:2135-2141.

[8] F. Digham, M. Alouini, and M. Simon, "On the energy detection of unknow signals over fading channels,” in Proc. IEEE International Conf. Commun, May. 2003, pp.3575-3579.

[9] Y. Chen, "Improved energy detector for random signals in Gaussian noise,” IEEE Trans. Wireless Commun, Feb. 2010, vol. 9, no. 2, pp. 558563.

[10] I. Gradshteyn and I. Ryzhik, Table of Integrals, Series, and Products, 5th edition. Academic Press, 1994. 